\title{
Lidil
}

Revue de linguistique et de didactique des langues

$36 \mid 2007$

Échanges exolingues via internet et appropriation des langues-cultures

\section{Les échanges exolingues via Internet}

Nouveau terrain d'exploration en didactique des langues

Christian Degache et François Mangenot

\section{OpenEdition}

\section{Journals}

Édition électronique

URL : http://journals.openedition.org/lidil/2373

DOI : $10.4000 /$ lidil. 2373

ISSN : 1960-6052

Éditeur

UGA Éditions/Université Grenoble Alpes

Édition imprimée

Date de publication : 1 décembre 2007

Pagination : 5-22

ISBN : 978-2-84310-108-3

ISSN : $1146-6480$

Référence électronique

Christian Degache et François Mangenot, «Les échanges exolingues via Internet », Lidil [En ligne], 36

2007, mis en ligne le 27 juin 2009, consulté le 21 septembre 2020. URL : http://

journals.openedition.org/lidil/2373; DOI : https://doi.org/10.4000/lidil.2373

Ce document a été généré automatiquement le 21 septembre 2020.

(c) Lidil 


\section{Les échanges exolingues via Internet}

Nouveau terrain d'exploration en didactique des langues

Christian Degache et François Mangenot

1 Les dispositifs ou environnements d'apprentissage sur Internet permettant à des groupes d'apprenants de développer des compétences en langues étrangères au moyen d'échanges en réseauavec des groupes d'apprenants alloglottes distants se sont multipliés depuis une dizaine d'années. Le projet eTandem, auquel fait écho ici même l'article de Lewis et Stickler, a ouvert la voie en rendant son serveur accessible aux groupes constitués. D'autres projets de "télécollaboration» ou d'« apprentissage en réseau » sont rapidement apparus ${ }^{1}$. Certains de ces projets feront l'objet des analyses présentées ici, d'autres seront seulement évoqués, y compris dans ce texte de présentation. De plus, les références données pourront permettre au lecteur de consulter des publications les abordant pleinement.

\section{Des initiatives multiples}

2 Le secteur des échanges en ligne entre groupes est très dynamique et s'inscrit, du point de vue des enseignants de langues, en particulier à l'école primaire et secondaire, dans la "pédagogie de l'échange à distance » (Maurice, 2004), donnant suite aux pratiques plus anciennes de correspondance et d'échanges scolaires, comme en témoigne le site Prim'langues. Plusieurs initiatives visent en outre à en étendre la pratique à d'autres domaines que celui des langues, traditionnellement plus engagé dans cette pédagogie (voir Carnet de route pour élaborer un projet d'échange à distance, sitographie). C'est le cas notamment de l'action européenne eTwinning ${ }^{2}$ qui, dans une perspective interdisciplinaire collaborative intégrant la dimension langagière (notamment à travers le choix de «la/les langue(s) de travail », Auboiron, 2005), encourage la mise en œuvre de partenariats scolaires à l'échelle européenne sur Internet. L'échange à distance entre groupes d'apprenants se caractérise toutefois par une profusion de désignations recouvrant ou non des différences de nature, ce qui nuit probablement à 
son unité : dialogues interclasses (PrimLangues), partenariat scolaire (eTwinning), partenariat de classes (Tele-Tandem, cf. Dausendschön-Gay, 2005: 13) ou encore partenariat collaboratif, mobilité virtuelle, jumelage électronique, projet multilatéral... Quoi qu'il en soit, cette variété témoigne d'expériences présentant une diversité d'objectifs, de contextes et de pratiques langagières, où la dimension interculturelle tient souvent une place importante et dont les dénominateurs communs sont de mettre en contact des groupes d'apprenants à travers leurs institutions, d'assurer un encadrement pédagogique aussi adapté que possible, et, pour celles qui se donnent des objectifs langagiers, de tabler sur les vertus de la communication authentique pour faciliter l'appropriation des langues-cultures visées.

\section{Filiations scientifiques}

3 En dépit du dynamisme du secteur, la recherche s'est encore peu intéressée aux phénomènes propres à ces situations d'appropriation et aux problématiques soulevées. L'analyse d'échanges en ligne à finalité langagière et culturelle peut s'appuyer sur différents ancrages théoriques, relevant de disciplines ou sous-disciplines diverses; il est sans doute utile de proposer une cartographie synthétique du territoire complexe des différents champs de référence possibles, même si certains de ces champs sont moins convoqués que d'autres par les auteurs de ce numéro.

De manière très large, tout d'abord, toute forme de communication humaine via Internet peut être considérée comme relevant de la Computer-Mediated Communication $(C M C)$, en français Communication médiatisée par ordinateur (CMO), champ de recherches dont l'objectif est d'analyser les nouveaux modes de communication induits par les outils informatiques. La CMC est solidement établie aux États-Unis, ce dont témoignent notamment l'existence et la notoriété du Journal of Computer-Mediated Communication fondé en 19953; on notera que cette revue se réclame des sciences sociales («Its focus is social science research on computer-mediated communication »). En France, la CMO concerne essentiellement des chercheurs en Sociologie (Flichy, 2001), en Sciences de l'information et de la communication (Marcoccia, 1998, 2003) et en Sciences du langage (Anis, 1998, en est l'un des pionniers). Des thématiques liées à la didactique des langues étaient présentes dans plusieurs manifestations scientifiques françaises récentes consacrées au domaine ${ }^{4}$. Une approche $\mathrm{CMO}$ des échanges en ligne peut mettre l'accent sur la dimension sémio-pragmatique de certains outils ou de certaines fonctionnalités (Mangenot, 2004, pour les forums pédagogiques), sur les variations langagières auxquelles donne lieu la communication électronique (Pierozak, 2003 et ici même) ou encore sur les nouveaux genres qui émergent à la faveur des situations de communication permises par les nouveaux outils (Mourlhon-Dallies, 2007).

Peut-on ensuite, à l'instar de Kern (2006), considérer que la CMO appliquée aux situations de formation devient simplement la "Communication pédagogique médiatisée par ordinateur»? Ce serait sans doute sous-estimer la spécificité des situations éducatives et faire fi d'autres chercheurs s'intéressant à la formation en ligne sous l'angle de la Formation ouverte et à distance (FOAD), du Computer-supported Collaborative Learning (CSCL) ou des Environnements informatiques pour l'apprentissage humain (EIAH). La FOAD est surtout investie par des chercheurs en Sciences de l'éducation et ses thématiques font une place importante aux questions d'autonomie, d'innovation, de dispositifs (Choplin et Galisson, 2002); la récente revue Distances et 
savoirs (Hermès) représente bien ce domaine en France. Elle s'intéresse par contre moins aux systèmes informatiques et à la manière dont ceux-ci facilitent ou au contraire contraignent les échanges en ligne et ne prend qu'occasionnellement pour objet l'analyse de ces échanges eux-mêmes. Le CSCL (ou les EIAH, en Frances), à l'inverse, fait une place importante à la technologie: des chercheurs en Psychologie cognitive, en Sciences de l'éducation (technologies éducatives) et en Informatique se penchent, depuis la fin des années 1980, sur «l'apprentissage dans le contexte d'activités collaboratives et les moyens d'encourager cet apprentissage à travers les technologies ${ }^{6} »$. Les chercheurs de ce domaine adoptent le plus souvent une démarche expérimentale : à la recherche de résultats mesurables, ils construisent la plupart du temps des dispositifs - voire des systèmes informatiques - ad hoc et cherchent à neutraliser certaines variables (socio culturelles ou socio affectives, notamment) qui, d'un point de vue didactique, sont pourtant incontournables. Cette tradition d'inspiration positiviste s'oppose aux traditions plus qualitatives, cherchant à observer, décrire et comprendre les logiques des acteurs, dans des situations écologiques, comme c'est le cas de tous les articles de ce numéro. On retrouve toutefois des préoccupations communes chez la plupart des chercheurs en technologies éducatives, qu'ils s'inscrivent dans une démarche expérimentale ou informatique (CSCL, EIAH) ou dans une approche plutôt descriptive (Didactique des langues) : il s'agit d'une part de la question des interactions verbales, dont l'importance dans la construction des savoirs et en tant que traces utilisables a posteriori par les chercheurs est reconnue, d'autre part de la question des tâches et des scénarios de communication (expression définie dans Mangenot et Zourou, ici même), qui doivent être conçus de manière à susciter les interactions les plus profitables; ces deux questions sont présentes chez la plupart des auteurs de ce numéro de Lidil.

Enfin, pour qui met spécifiquement l'accent sur les apports des échanges via Internet à l'apprentissage/appropriation des langues, la didactique des langues (ou la Second Language Acquisition Research des Anglo-Saxons) a déjà produit un important corps de recherches concernant les échanges exolingues (voir plus loin), les interactions en classe de langue (Cicurel et Bigot, 2005, pour une revue récente) et les questions liées au développement de la compétence culturelle (voir les articles de ce numéro qui citent les principales sources de ce domaine). Il est intéressant de noter qu'aux États-Unis un courant de recherche combinant l'usage d'Internet, les interactions en ligne et l'interculturel a récemment vu le jour, baptisé Internet-mediated Intercultural Foreign Language Education (ICFLE, Belz et Thorne, 2006); on y retrouve un certain nombre d'acteurs connus du domaine de l'apprentissage des langues assisté par ordinateur (CALL), mais également des chercheurs a priori non spécialistes des TICE, comme Claire Kramsch (Kramsch \& Thorne, 2002). La plupart des articles de ce numéro de Lidil, pourtant planifié avant que ne soit publié l'ouvrage américain, pourraient venir se ranger sous cette dénomination, la question du développement de la compétence culturelle y étant très présente, et ils pourraient sans doute reprendre à leur compte les arguments par lesquels Thorne (2006:3) justifie l'acronyme ICFLE :

- les désignations utilisées jusqu'à présent, comme « télécollaboration », ne mentionnaient pas la dimension culturelle ;

- «Foreign Language Education » renvoie spécifiquement d'une part à la didactique des langues en tant que telle, d'autre part à la notion humaniste d'éducation. 
7 On terminera ce tour d'horizon en signalant quelques revues ou colloques récents ayant abordé la question des échanges pédagogiques en ligne dans le domaine des langues-cultures et en tentant de situer ce numéro de Lidil par rapport à ses prédécesseurs. À notre connaissance, la première revue à avoir consacré un numéro entier à ce thème est Language Learning \& Technology, sous le titre "Telecollaboration » (Belz, 2003) ; dans sa présentation du numéro, la coordinatrice (Belz, $2003: 2)$ propose une définition qui nous semble toujours actuelle :

[...] telecollaboration is characterized by institutionalized, electronically mediated intercultural communication under the guidance of a languacultural expert (i.e., a teacher) for the purposes of foreign language learning and the development of intercultural competence.

8 On a vu plus haut que Belz s'était maintenant rangée sous l'étiquette ICFLE. La même année paraissait le numéro 28 de la revue Lidil, intitulé "Intercompréhension en langues romanes » (Degache, 2003), pour moitié consacré à la thématique des échanges en ligne mais focalisé sur un seul dispositif, Galanet; on retrouvera des analyses concernant celui-ci dans trois articles du présent numéro, dans le prolongement des problématiques alors abordées, notamment sur la catégorisation des phénomènes de nature interculturelle et la description des représentations en jeu dans les rencontres générées par ce dispositif, et surtout sur les conditions de leur intégration locale au niveau d'un groupe. On a déjà mentionné l'ouvrage de Belz et Thorne (2006), actes du colloque 2005 de l'American Association of University Supervisors, Coordinators and Directors of Foreign Language Programs; les projets analysés sont de natures diverses, l'accent étant mis dans plusieurs chapitres sur la question de la communication ratée (" missed communication ») ou encore sur celle de la nécessaire prise en compte des usages technologiques («cultures-of-use of Internet communication tools », Thorne, 2003). La revue américaine Calico Journal ${ }^{7}$ a publié pour sa part en 2006 un numéro intitulé «What does it take to teach on line?»; comme ce titre l'indique, la problématique était centrée sur le rôle des enseignants-tuteurs, alors que cette livraison de Lidil met plus l'accent sur les apprenants. La même année, la revue Le Français dans le monde, Recherches et applications a consacré un numéro aux "Échanges en ligne dans l'apprentissage et la formation » (Dejean-Thircuir et Mangenot, 2006) ; neuf articles sur quatorze traitent de situations d'apprentissage des langues, les autres étant consacrés à la formation des enseignants; mais on constate que l'approche interculturelle n'est convoquée que par un seul article alors que la question de l'influence des outils technologiques est beaucoup plus présente (sept articles). On peut enfin signaler le récent colloque « Échanger pour apprendre en ligne » (EPAL), dont environ un tiers des communications (17 sur 50) ont porté sur l'apprentissage des langues en réseau ; treize d'entre elles sont disponibles dans les Actes en ligne du colloque (Lamy, Mangenot et Nissen, 2007) et certaines paraitront en 2008 dans la revue Alsic. Toutes les thématiques mentionnées plus haut sont abordées par l'un ou l'autre de ces treize articles, de l'influence des technologies au développement de compétences culturelles, en passant par le rôle des tuteurs et par la question des dispositifs hybrides.

9 Ce numéro de Lidil se penche sur certaines des problématiques évoquées et sur les retombées de ce type d'échanges en ligne du point de vue de la didactique des langues, en privilégiant les interactions entre groupes d'apprenants alloglottes sur trois plans :

- au plan interactionnel, comment la dimension interculturelle est-elle prise en charge dans ces échanges? S'agit-il seulement de confronter et/ou comparer des points de vue et des 
pratiques culturels différents ou bien cette dimension est-elle également en jeu, sur le plan pragmatique, à travers les échanges, dans le cadre par exemple de décisions à prendre pour un travail collaboratif? Dans un cas comme dans l'autre, quels en sont les effets sur la situation d'apprentissage?

- au plan de la méthodologie d'enseignement, comment et avec quels résultats les échanges sont-ils mis à profit au niveau du/des groupe(s) pour développer la compétence à communiquer langagièrement?

- au plan logistique et organisationnel, comment et dans quelles conditions ces échanges sontils mis en œuvre en réseau entre les groupes ? Comment et dans quelle mesure influencentils l'organisation et les pratiques du groupe au niveau local ?

On notera que l'appel à contribution prévoyait un volet centré sur la dimension technologique, ainsi formulé: "Quel est l'impact des outils et des canaux de communication utilisés : outils synchrones ou asynchrones, canal exclusivement écrit ou également oral, rôle éventuel de l'image, intérêt de disposer d'une plateforme dédiée? ». Mais aucun auteur, à l'exception, dans une certaine mesure, de Pierozak, n'a suivi cette piste, plus présente par contre dans Dejean-Thircuir et Mangenot (2006) et dans le colloque EPAL (cf. supra).

\section{L'échange exolingue en question}

Comme en témoigne le titre de ce numéro, l'échange exolingue constitue, dans les situations de communication qui seront considérées ici, une notion centrale qu'il convient d'interroger compte tenu des traits particuliers qu'elle y revêt. Nous pouvons en identifier quatre : (i) il s'agit d'un échange en ligne sur Internet, (ii) il est tout à la fois le stimulateur et le vecteur de l'appropriation, (iii) il est rarement établi entre deux personnes seulement mais concerne en règle générale un collectif d'individus, (iv) il peut avoir lieu dans une seule langue, dans deux ou plus encore.

Toutes les initiatives mentionnées plus haut réunissent des interactants avec des profils de compétences linguistiques asymétriques, ce qui constitue bel et bien la principale caractéristique de l'échange exolingue. Si l'échange exolingue est considéré en linguistique de l'acquisition et en didactique des langues comme une communication établie "par des moyens autres qu'une langue maternelle éventuellement commune aux participants » (Porquier, $1984: 18$ ), il est fréquemment rappelé en effet que cet échange se caractérise par une "asymétrie dans l'accès des interlocuteurs aux différents codes de l'interaction » (Matthey et De Pietro, 1997) qu'ils assument consciemment comme un facteur déterminant la nature des interactions à travers une "thématisation récurrente de cette asymétrie - surtout apparente lors de la résolution de difficultés (malentendus, incompréhensions, etc.) » (Py, 1990: 82). Or, si la plupart des études ont été conduites, le plus souvent pour des «raisons d'opportunité » (Py, ibid.), autour d'échanges entre natifs et non-natifs dans une seule langue, et bien que le projet initial de Porquier l'envisageât ${ }^{8}$, peu d'études ont considéré les échanges exolingues basés sur l'utilisation négociée de plusieurs langues (deux, trois, voire davantage). C'est pourtant bien ce qui caractérise la plupart des projets tablant sur les interactions en ligne entre groupes d'apprenants hétéroglottes. Se configurent entre eux des situations de contact actualisant différents types d'échanges exolingues en fonction du nombre de langues impliquées dans l'échange. À partir de là, les échanges exolingues générés par ces tâches peuvent être soit monolingues, par le 
recours à une langue de communication, soit bilingues, soit plurilingues, et faire l'objet d'un « contrat codique » (Cambra Giné, 2003 ; Degache, 2006).

Les contributions présentées dans ce numéro de Lidil témoignent précisément de la diversité de ces types d'échanges que l'on regroupera en trois catégories en s'inspirant de la typologie des interactions verbales présentée par Matthey et De Pietro ${ }^{9}$ (1997):

1. échanges exo-monolingues : Mangenot et Zourou (projet Le Français en $1^{\text {re }}$ ligne);

2. échanges exo-bilingues : Audras et Chanier (projet tridem en anglais et français); Dolci et Spinelli (projet Incontro en italien et anglais); Lewis et Stickler (projet Tandem en anglais et allemand);

3. échanges exo-plurilingues: Araújo e Sá, Ceberio et Melo; Carrasco et Pishva ; Degache, López et Séré (projet Galanet en langues romanes, notamment en espagnol, français, italien et portugais).

L'article de Pierozak, parce que portant sur des interactions exo-monolingues interindividuelles hors de tout contexte pédagogique, viendra souligner en contrepoint les spécificités de ces échanges exolingues à finalité d'apprentissage.

Signalons toutefois que le nombre de langues impliquées dans l'échange n'est pas le seul critère de caractérisation. Dès lors que deux langues ou plus sont impliquées dans l'échange exolingue, encore faut-il considérer selon quelles modalités « contractuelles » elles le sont : chaque participant utilisera-t-il la langue de son choix, la langue qu'il maitrise le mieux, la langue d'un autre interlocuteur parce qu'il la cible en priorité ? Son choix sera-t-il fixe ou bien variera-t-il en fonction des circonstances, instrumentalisant ainsi l'«alternance codique", y compris en ayant recours ponctuellement ou fréquemment à une langue tierce? Ces questions toutefois, ne seront considérées ici que de façon incidente, au fil des problématiques abordées. D'autres développements seront nécessaires à l'avenir pour mieux décrire et caractériser ce secteur des échanges exolingues en ligne.

\section{Présentation des articles}

16 Les huit contributions présentées dans ce numéro s'appuient toutes sur des usages " écologiques », non suscités à des fins d'expérimentation et sans contrôle de variables, en s'employant à décrire le dispositif qui est à l'origine des échanges et à caractériser le type de communication qui s'y déroule. Les données prises en compte dans ces différents articles, de natures très diverses, sont issues d'une ou plusieurs des catégories suivantes :

- des données réflexives tirées de questionnaires, d'écrits rétrospectifs, d'entretiens individuels ou à plusieurs, auprès des apprenants mais aussi parfois des enseignants ;

- les produits de différentes tâches, individuelles (profils) ou collaboratives (pages web) ;

- des interactions au sein d'un seul groupe en présentiel ou à distance (messages instantanés) ;

- et surtout, des interactions en ligne entre groupes de différents types : courriels, clavardages, forums, blogs, oral synchrone.

Si l'on examine les problématiques abordées, on note tout d'abord la prédominance de la question du développement de la compétence culturelle, question centrale dans les cinq premiers articles de ce numéro, alors qu'il ne s'agissait que de l'une des quatre pistes de réflexion proposées initialement. Il n'y a pas lieu de s'en étonner car on y voit de longue date le principal intérêt des échanges exolingues via Internet (voir notamment les références américaines supra). Comme le dit Porcher (2002) dans la 
conférence inaugurale d'un colloque sur l'ouverture internationale et les échanges, «ce n'est pas le plurilinguisme qui est vraiment en cause, c'est le pluriculturalisme, c'est-àdire la capacité de comprendre les valeurs de l'autre et de comprendre ses pratiques culturelles ». Cet enjeu est d'ailleurs bien présent dans la nature même de l'échange exolingue, dont on sait que «l'asymétrie linguistique est généralement prolongée par des asymétries dans les règles de l'interaction et les conventions culturelles » (Py, $1990: 82$ ).

Outre le fait de s'intéresser sous différents angles à ces asymétries culturelles entre groupes interagissant sur Internet, ces cinq articles ont également en commun, sur le plan méthodologique, de chercher à mettre au point des procédures de caractérisation qualitative des échanges observés. Ce faisant, il devient possible de rendre compte de phénomènes allant au-delà de la simple prise de contact avec l'autre.

Audras et Chanier visent à établir des critères permettant de juger de l'authenticité de la rencontre interculturelle à l'intérieur de «tridems » de nationalités différentes (États-Unis, Royaume-Uni et France) et d'apprécier le développement de la compétence interculturelle à l'aune des dimensions de prises de risque ou d'engagement chez les participants. Mangenot et Zourou, en relation avec la piste méthodologique prévue dans l'appel à communication (cf. supra), font ressortir le rôle des tâches dans le déclenchement de "rich points " provoquant une prise de distance par rapport à sa propre culture et une réflexion sur la culture de l'autre; ils se fondent sur deux situations tirées du projet "Le français en (première) ligne ", qui met en relation des étudiants en master FLE avec des apprenants de divers pays (ici, des Australiens et des Japonais). Dolci et Spinelli, dans le cadre d'un projet italo-américain, abordent la question du travail collaboratif en groupes restreints et de l'idioculture qui se crée à l'intérieur de chaque groupe, celle-ci étant vue à la fois comme composée - pour partie - d'éléments provenant des deux cultures en contact et comme nécessaire à la cohésion du groupe. On pourra tenter de rapprocher leur notion d'idioculture de la notion de «vie de groupe » développée par Audras et Chanier. De même, on pourra examiner, chez les auteurs de ces trois articles, si le sens donné aux expressions «incident critique ", " rich point » ou " élément déclencheur » recèle des nuances ou recouvre une même réalité. Degache, López et Séré, pour leur part, abordent la question de l'interculturalité dans ces échanges de manière plus générale, cherchant à repérer et à catégoriser les manifestations interculturelles à l'intérieur du dispositif Galanet orienté vers l'intercompréhension en langues romanes. Au sein de leur appréciation de la place et $\mathrm{du}$ fonctionnement des échanges thématisant l'interculturalité, la question des conflits est également considérée. Dans le même dispositif, Araújo e Sá, Ceberio et Melo s'intéressent aux représentations sur les langues telles qu'elles se font jour initialement dans les profils personnels complétés par les participants et telles qu'elles évoluent ensuite dans l'interaction synchrone écrite.

Ces cinq premiers articles, en dépit de la diversité des situations de communication considérées et en empruntant des approches et des terminologies différentes, mettent en évidence que la rencontre interculturelle n'est pas une donnée constitutive de l'interaction exolingue mais résulte d'une construction collaborative entre les participants qui décident ou non d'introduire cette dimension dans l'échange. D'autres points de convergence pourront être identifiés par le lecteur, comme par exemple la distinction entre culture partagée, culture divergente et culture commune en construction, ou encore, sur le plan didactique en relation avec le deuxième volet de 
l'appel à contribution (cf. supra), l'effet de l'incitation à la comparaison dans la consigne de la tâche ou dans les choix des étudiants et la relation entrescénario et construction des compétences interculturelles.

21 Les problématiques abordées dans les trois autres articles portent sur d'autres aspects que l'interculturalité. Si tous les dispositifs décrits dans ce numéro comportent une dimension hybride, combinant le plus souvent des échanges exolingues à distance et des séances en présentiel plutôt monolingues conduisant soit à préparer les échanges, soit à y revenir de manière réflexive, seul l'article de Carrasco et Pishva thématise cette dimension en accord avec la troisième orientation problématique du numéro, en s'interrogeant sur la meilleure manière pour les tuteurs de jouer sur ces deux tableaux, notamment en termes de délivrance des consignes. Adoptant pour sa part l'angle de l'étudiant, dans le cadre de eTandems, l'article de Lewis et Stickler aborde la question des stratégies mises en œuvre par les apprenants pour parvenir à une communication exolingue satisfaisante; la typologie bien connue de Rebecca Oxford est convoquée mais les auteurs font également ressortir des stratégies spécifiques à la communication électronique. Le seul article qui ne s'appuie pas sur un contexte pédagogique institutionnel est celui de Pierozak: celle-ci examine, de manière exploratoire, des situations de communication électronique exolingues faisant usage de divers dispositifs (IRC, MSN messenger, courriel, SMS) ; s'agissant de locuteurs résidant en France, une des questions sous-jacentes est alors celle de la spécificité de cette communication électronique par rapport à des situations en face-à-face.

$\mathrm{Au}$ vu du développement des projets et des recherches qui y sont associées, nul doute que cette question des réseaux de groupes en ligne constitue désormais un nouveau terrain d'exploration pour la didactique des langues. Même si on ne mesure encore pas bien les retombées pour celle-ci, on peut s'attendre à ce qu'elles soient importantes, notamment parce que ces réseaux génèrent un type d'interactions échappant à certaines distinctions structurantes dans cette discipline depuis une trentaine d'années, comme par exemple l'opposition entre interactions sociales en milieu naturel et interactions en classe de langue en milieu dit institutionnel ou encore l'opposition entre appropriation en milieu alloglotte et en milieu homoglotte. C'est toute l'ambition du présent volume, comme on pourra en juger à la lecture des différentes contributions et des problématiques traitées, que de vouloir contribuer à cette exploration.

\section{BIBLIOGRAPHIE}

\section{Références bibliographiques}

Anis, J. (1998) : Texte et ordinateur : l'écriture réinventée ? Bruxelles, De Boeck Université.

Auboiron, P. (2005) : Action européenne eTwinning, Partenariats scolaires en Europe, Actes du colloque « Cyberlangues 2005 » (voir sitographie).

Belz, J.A. (2003, dir.) : Language Learning \& Technology 7 (2), Telecollaboration, revue en ligne. 
Belz, J. A., Thorne, S. (2006, dir.) : Internet-Mediated Intercultural Foreign Language Education, Boston, Thomson Heinle.

Cambra Giné, M. (2003) : Une approche ethnographique de la classe de langue, Collection LAL, Paris, Didier.

Choplin, H. et Galisson, A. (2002, dir.) : Éducation permanente, 152, Les TIC au service des nouveaux dispositifs de formation.

Cicurel, F. et Bigot, M. (2005, dir.) : Le Français dans le monde, Recherches et applications, 38, Les interactions en classe de langue.

Dausendschön-Gay, U. (2005) : Introduction, Mon cahier Tele-Tandem, OFAJ, Paris-Berlin.

Dausendschön-gay, U., Krafft, U. (1998) : Quand l'exolingue devient de l'interculturel, Lidil, 18, Alternance des langues : enjeux socioculturels et identitaires, 92-111.

Degache, C. (2003, dir.) : Lidil, 28, Intercompréhension en langues romanes.

Degache, C. (2006) : Aspects du contrat didactique dans une formation plurilingue ouverte et à distance, Le Français dans le monde, Recherches et applications, 40, 58-74.

Dejean-Thircuir, C., Mangenot, F. (2006, dir.) : Le Français dans le monde, Recherches et applications, 40, Les échanges en ligne dans l'apprentissage et la formation.

Flichy, P. (2001) L'imaginaire d'Internet, Paris, La Découverte.

Kern, R. (2006) : La communication médiatisée par ordinateur en langues : recherches et applications récentes aux USA, Le Français dans le monde, Recherches et applications, 40, 17-29.

Kramsch, C., Thorne, S. (2002) : Foreign Language Learning as a Global Communicative Practice, in D. Block, D. Cameron (dir.) Globalization and Language Teaching, 83-100, London, Routledge.

Lamy, M.-N., Mangenot, F., Nissen, E. (2007, dir.) : Actes du colloque «Échanger pour apprendre en ligne» (EPAL), en ligne.

Mangenot, F. (2004) : Analyse sémio-pragmatique des forums pédagogiques sur Internet, Salaün, J.-M. \& Vandendorpe, C. (dir.), Les défis de la publication sur le Web : hyperlectures, cybertextes et métaéditions, 103-123, Villeurbanne, Presses de l'Enssib.

Marcoccia, M. (1998) : La normalisation des comportements communicatifs sur Internet : étude sociopragmatique de la netiquette, in N. Guéguen \& L. Toblin (éds.), Communication, société et Internet, Paris, L'Harmattan, 15-22.

Marcoccia, M. (2003) : Parler politique dans un forum de discussion, Langage \& Société 104, 9-55.

Matthey, M., De Pietro, J.-F (1997) : La société plurilingue : utopie souhaitable ou domination acceptée ? Plurilinguisme : « contact » ou « conflit » de langues ? L'Harmattan, 133-190.

Maurice, M. (2004) : " L'échange n'est pas une situation banale, ne la banalisons pas ", Congrès national des professeurs de français, Lisbonne, 2004, consulté le 1-9-2007.

Mourlhon-Dallies, F. (2007) : Communication électronique et genres du discours, Glottopol, 10, 11-23, revue en ligne.

Pierozak, I. (2003) Le « français tchaté » : un objet à géométrie variable ? Langage \& société, 104, 123-144.

Pierozak, I. (2007, dir.) : Glottopol, 10, Regards sur l'internet, dans ses dimensions langagières. Penser les continuités et discontinuités, revue en ligne. 
Porcher, L. (2002) : Pour une identité plurielle, Conférence inaugurale, Université d'automne « Ouverture internationale et échanges : pour une pédagogie raisonnée des différences ", CIEP/ OFAJ, Sèvres, oct. 2002, consulté le 1-9-2007.

Porquier, R. (1984) : Communication exolingue et apprentissage des langues, Acquisition d'une langue étrangère III, Paris, Presses universitaires de Vincennes ; Neuchâtel, Centre de linguistique appliquée, 17-47.

Py, B. (1990) : Les stratégies d'acquisition en situation d'interaction, D. Gaonac'h (coord.), Le français dans le monde, Recherches et applications, Acquisition et utilisation d'une langue étrangère. L'approche cognitive, Paris, Hachette, 81-88.

Reboul-touré, S., Mourlhon-dallies, F., Rakotonoelina, F. (2004, dir.), Les Carnets du Cediscor, 8, Les discours de l'internet, nouveaux corpus, nouveaux modèles?

Thorne, S. (2003) : Artifacts and Cultures-of-Use in Intercultural Communication, Language learning \& Technology 7 (2), Telecollaboration, 38-67, revue en ligne.

Thorne, S. (2006) : Pedagogical and Praxiological Lessons from Internet-mediatedIntercultural Foreign Language Education Research, J.A. Belz et S. Thorne, 2-30.

Sitographie (liens vérifiés le 1-9-2007)

\section{Colloques}

« La communication électronique : Approches linguistiques et anthropologiques », Paris, février 2004.

CSCL 2005 (Taiwan)

Cyberlangues 2005

«La langue de la communication médiatisée par les technologies de l'information et de la communication (CMT) », Bordeaux, mai 2006.

«Échanger pour apprendre en ligne » (EPAL), Grenoble, juin 2007.

\section{Revues scientifiques}

$\underline{\text { Alsic }}$

Computer-assisted Language Instruction Consortium Journal (sommaires et résumés de la revue)

Glottopol

Journal of Computer-Mediated Communication

Language Learning \& Technology

Projets, aide aux projets

Carnet de route pour élaborer un projet d'échange à distance (d'après M. Maurice)

Cultura

eTandem

eTwinning

Galanet

Incontro (Italien, anglais)

Le français en (première) ligne 
Lingalog

Literalia

$\underline{\text { Penn State FL Telecollaboration Project }}$

$\underline{\text { Prim'langues }}$

Tele-tandem

\section{NOTES}

1. Comme par exemple les projets Cultura, Tele-tandem, Penn State Foreign Language Telecollaboration Project, Progetto Incontro, tridem, Le français en (première) ligne, Galanet, Lingalog, Literalia (voir sitographie en fin d'article).

2. Opération pilote mise en œuvre en 2005 par la Direction Éducation et culture de la Commission européenne dans le cadre du programme " e-learning » et désormais intégrée dans le programme d'éducation et formation tout au long de la vie (Lifelong Learning Programme 2007-2013). Cette opération est dans certains états de l'Union européenne relayée au niveau local par un programme spécifique comme c'est le cas en Belgique avec l'action eJumelage-eTwinning dans la Communauté française.

3. Rédactrice en chef : Susan Herring. Quatre numéros par an. Revue en ligne. Certains numéros ou articles ont été consacrés à des problématiques de formation en ligne.

4. Colloques «La communication électronique : Approches linguistiques et anthropologiques ", 5-6 février 2004, Paris, "La langue de la communication médiatisée par les technologies de l'information et de la communication (CMT) ", Bordeaux, 2006 (voir Sitographie). Revues Carnets du Cediscor, 8 (Reboul-Touré et al., 2004), Glottopol, 10 (Pierozak, 2007).

5. Le domaine $E I A H$ est proche du CSCL, quoique plus investi par les informaticiens et donc plus préoccupé par le développement de systèmes informatiques.

6. Selon le site du dernier colloque mondial CSCL, à Taiwan (notre traduction).

7. Computer-assisted Language Instruction ConsortiumJournal (voir Sitographie).

8. Soulignant la diversité et l'hétérogénéité de la communication exolingue (1984:23), Porquier ébauchait dans cet article une « esquisse de typologie de situations exolingues » (ibid. : 29).

9. Typologie mise au point à partir d'une « réflexion sur les types et les fonctions des marques transcodiques" en considérant deux axes: l'axe unilingue-bilingue (rendant compte de la disponibilité éventuelle d'une $2^{\mathrm{e}}$ langue dans le répertoire verbal des interlocuteurs) et l'axe endolingue-exolingue (rendant compte du degré de partage du ou des code(s) par les interlocuteurs, ou degré d'asymétrie). Les auteurs identifient ainsi quatre situations prototypiques: unilingueendolingue (asymétrie linguistique minimale dans une seule langue); unilingue-exolingue (asymétrie linguistique patente, prise en charge par les interlocuteurs, dans une seule langue); bilingue-endolingue (asymétrie minimale dans deux langues, ou parler bilingue) et exolingue-bilingue (deux langues inégalement maitrisées sont utilisées, les rôles impliqués par les divergences codiques pouvant s'inverser). 


\section{AUTEURS}

\section{CHRISTIAN DEGACHE}

Université Stendhal-Grenoble3, Lidilem, E. A. 609

\section{FRANÇOIS MANGENOT}

Université Stendhal-Grenoble3, Lidilem, E. A. 609 